\title{
Repensar los movimientos sociales
}

DOI: https://doi.org/10.32870/espiral.v21i60.294

A lo largo de su vida académica Jorge Alonso ha producido una extensa bibliografía que lo coloca como uno de los antropólogos con mayor obra escrita en nuestro país. Campea en los textos de Jorge Alonso el conocimiento de los temas abordados y un enfoque crítico que, de hecho, es característico de su actitud como antropólogo. En su más reciente libro, Repensar los movimientos sociales, Jorge Alonso elabora una revisión exhaustiva de la bibliografía analítica que trata el tema.

Con prosa reflexiva, a lo largo de una introducción, cuatro capítulos y un apéndice, Jorge Alonso examina los distintos enfoques y la variedad de opiniones que se han escrito sobre movimientos sociales, especialmente desde la segunda mitad del siglo XX. Al final de su obra y a título de apéndice, el autor incluye la reseña de Agrietar el capitalismo, de John Halloway (2011). Repensar... también está conformado por los escritos de Rafael Sandoval Álvarez y María Bertely Busquets, quienes a título de prefacio y prólogo nos ofrecen dos textos interesantes y útiles, que sitúan en contexto la presente obra la trayectoria académica de Alonso y las discusiones suscitadas por su punto de vista respecto a los movimientos sociales.

En la introducción Jorge Alonso relata al lector cómo fue que se interesó por el
Andrés Fábregas Puig*

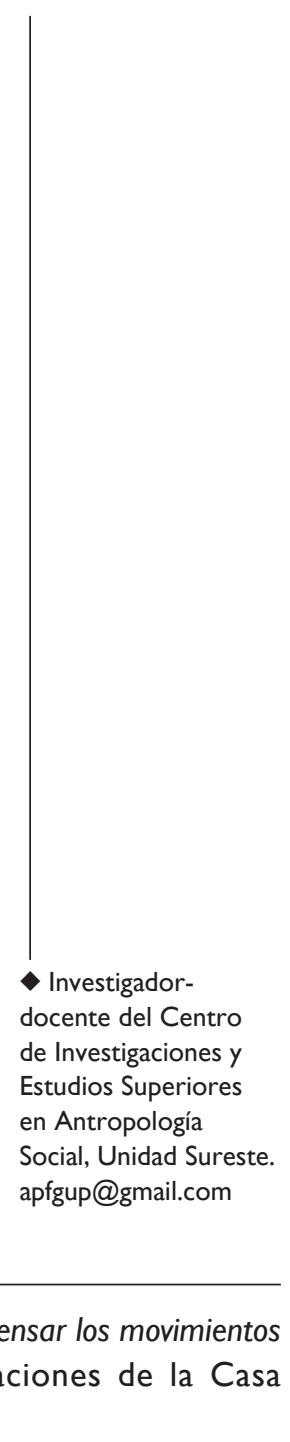

Jorge Alonso, 2013, Repensar los movimientos sociales, CIESAS/Publicaciones de la Casa Chata, México. 
análisis de los movimientos sociales a partir de sus primeras experiencias dirigiendo trabajo de campo en la Ciudad de México (en Coyoacán) en la década de los años sesenta, prolíficos en expresiones sociales de descontento y disidencia. Por supuesto, en Repensar los movimientos sociales Jorge Alonso no se remonta a esas primeras experiencias como estudioso del tema, pues su objetivo es invitar a otros a pensar el tema desde los planteamientos y discusiones recientes. Para ello, inicia con una serie de debates y reflexiones que han concurrido en un seminario plural a lo largo de más de cuatro años. Desde las primeras líneas, Alonso advierte al lector sobre su rechazo a las visiones esencialistas de los movimientos sociales, así como sobre su convicción de que estos son un tema abierto.

En el capítulo primero de Repensar los movimientos sociales, reconoce la existencia de un amplio abanico de perspectivas y opiniones, comentándolas con precisión. "Las teorías también se mueven", escribe Alonso (p. 34), tal como los mismos movimientos que tratan de explicar. Esta situación amerita que se realice una revisión sistemática de las teorías mismas, pues hay algo de lo que no pueden escapar ni los movimientos sociales ni las teorías: la condición humana.

Alonso se detiene en pensar en la propuesta de Alain Touraine (pp. 34-7) y la contrasta con las de Boaventura de Sousa Santos (pp. 37-41), tan leído en estas últimas décadas. Así, compara una visión elaborada desde una perspectiva eurocéntrica, con un giro hacia la dimensión cultural (Touraine), con una surgida de la observación de Latinoamérica (de Sousa Santos), más apegada a la reflexión sobre las relaciones sociales. La exigencia de los movimientos sociales -dice de Sousa Santos- está puesta en cambios concretos, impulsados por acciones colectivas. Desde las advertencias sobre el epistemicidio - que no escapa a la atención de Jorge Alonso- de Sousa Santos aboga por una concepción multi- 
cultural de los derechos humanos, como un ámbito nuevo en los movimientos sociales.

Pero todo lo anterior exige pensar en la complejidad de los movimientos mundiales alternativos. Existe un movimiento altermundista surgido como respuesta a las modalidades actuales del capitalismo neoliberal y su perjuicio en los territorios neocoloniales. Las formas de poder, entre las que resalta el Estado, responden a los intereses de una clase capitalista mundial, y al ser esto así, me parece que los movimientos sociales adquieren perspectivas más radicales cuando se dirigen no a reformar las instancias de poder, incluidos los Estados, sino a superar las relaciones sociales que impiden el ejercicio de la libertad, con todo lo que ello implica. No es el Estado en sí el objetivo, sino las relaciones sociales que sostienen el andamiaje del capitalismo neoliberal.

El primer capítulo incita a pensar en lo anterior y en los análisis que enfatizan el contrapoder o, incluso, el fin del poder (Nahim, 2013). Lo medular, me parece, es que un movimiento social se plantee la erradicación de las relaciones sociales -con sus respectivas consecuencias- que sostienen a la economía capitalista contemporánea. En esta tesitura, este primer capítulo es notable por la variedad de puntos de vista analizados y por la posibilidad que esto abre a pensar en alternativas. No es posible no coincidir con la afirmación de Jorge Alonso cuando sostiene que no existe una sola aproximación a los movimientos sociales, sino una gama amplísima de ellas.

En el segundo capítulo, intitulado "Movimientos sociales y convergencias", el autor examina las discusiones sobre el poder y las que atañen a las convergencias. En alusión a planteamientos como los de Touraine, Alonso destaca que en América Latina los movimientos sociales plantean soluciones concretas a problemas específicos que han surgido en una vida real de carencias. Alonso reflexiona las trayectorias de los movimientos anticapitalistas, la territorialización como 
cimiento de la alteridad de los movimientos sociales, y así se aproxima a una discusión sobre el poder y las dificultades que se presentan cuando se discute el contexto "comunitario" del poder o la concepción del "contrapoder"; en verdad, una discusión complicada, que el propio Carlos Marx asumió al criticar severamente lo que él llamó "la visión idúlica" de la comunidad y al escribir de la opresión del individuo a manos de la propia comunidad (Marx, 1968). ¿Cuáles son los poderes alternativos que garantizan las vías hacia la libertad? La respuesta está en el aire, parafraseando a Bob Dylan.

La discusión sobre las convergencias de los movimientos sociales anticapitalistas necesariamente orilla a reflexionar sobre la notable fragmentación y dispersión de los movimientos sociales. ¿Qué es lo que potencialmente podría unificar a los movimientos anticapitalistas actuales? Los tiempos del planteamiento de aquella "clase universal" que con su sola acción lograría el cambio del mundo parecen lejanos. Es por eso que coincido con Jorge Alonso en que la cuestión de las convergencias es prioritaria para aquella reflexión que busque encontrar de qué forma la pluralidad de movimientos sociales anticapitalistas pueden conjuntarse. Las propias estrategias de mundialización del capital hacen compleja la elaboración y puesta en práctica de una respuesta unificada. Mientras tanto, los propios procesos de convergencia constituyen, por el momento, la respuesta más alentadora. En ese sentido, Alonso reflexiona sobre experiencias concretas, como la del Foro Social Mundial (p. 83), uno de cuyos líderes intelectuales es Boaventura de Sousa Santos. Converger sin suprimir la pluralidad es uno de los desafíos más importantes. Sin duda, la reflexión sobre las convergencias, en el sentido que las plantea Alonso, es estratégica para los movimientos sociales anticapitalistas.

En el capítulo tercero Jorge Alonso recuenta y describe los movimientos sociales más destacados de México, no sin ofrecernos su análisis a propósito. El inventario sigue un 
Repensar los movimientos sociales

orden histórico que revela el endurecimiento continuo del Estado y del Capital hacia estos movimientos, así como la represión con que ambas entidades han respondido, invariable, a sus demandas. Aunado a lo anterior, vemos cómo la corrupción de los círculos de poder alcanzó niveles insospechados que la colocaron como una característica definitoria del llamado sistema político mexicano. El movimiento del Ejército Zapatista de Liberación Nacional marcó un momento de renovación de los movimientos sociales anticapitalistas en el país, sin olvidar otros movimientos que continúan en el escenario nacional. La erudición de Jorge Alonso se despliega con fluidez, haciendo observaciones precisas y reflexiones oportunas. No deja de ser notable que este repaso de los movimientos sociales en México se logre con brevedad. Sus páginas invitan a releer a historiadores como Eric Thompson, Chistopher Hill o Eric Hosbawm, autores sugerentes en la búsqueda de respuestas a los desafíos contemporáneos. Asimismo, existe en este capítulo -como de hecho, en todo el libro- una vocación comparativa que permite examinar a los movimientos sociales mexicanos en el contexto de Latinoamérica.

En el cuarto apartado Jorge Alonso discurre en torno a la experiencia del Seminario sobre Movimientos Sociales al que él mismo pertenece y que se ha celebrado en Jalisco desde el año 2007. Por su pluma nos enteramos de la composición plural del mismo, lo cual constituye uno de sus rasgos más notables. No es un seminario conformado exclusivamente por académicos: en su composición interviene una compleja variedad de personas y organizaciones provenientes de varias partes del estado de Jalisco. El seminario es un ejemplo de un proceso de convergencias, descrito como un amplio escenario de debates anticapitalistas que llevan la impronta de lograr sujetos antisistemáticos. La forma en que está narrado el capítulo revela al antropólogo acucioso que realiza observación participante y 
dialoga con otros protagonistas del mismo escenario, para lograr la ponderación buscada. La huella histórica es una constante en la escritura de Jorge Alonso, y está presente en "Colectivos que estudian otros movimientos" (pp. 100-5), "Colectivos que piensan y actúan desde la perspectiva del sujeto" (pp. 105, 106) y en "Los colectivos y el caminar preguntando" (pp. 106-8), desde donde llega al tema de "La búsqueda de la Autonomía" (pp. 108-10). Alguien que busque respuestas acabadas o formularios para la acción, quedará decepcionado: nada de ello hay en estas páginas; pero quien busque estímulos al pensamiento, encontrará una notable riqueza en ellas. Alonso nos proporciona un panorama de qué es lo que se plantean los colectivos en una amplia gama de temas y situaciones. En la sección que cierra el cuarto capítulo, titulada "Los colectivos, la resonancia, las vinculaciones y las convergencias" (pp. 110-2), el autor abunda en cómo enlazar esas dimensiones, examinando qué piensan los colectivos acerca de sí mismos.

En "Hacia algunas conclusiones" (pp. 113-7) escribe insistiendo en la variedad de los movimientos sociales y las formas colectivas en las que se apoyan. Resalta la resistencia a la cooptación -tan practicada por el Estado mexicano-, aboga por el análisis de las formas políticas con perspectivas históricas y no esencialistas, advierte la importancia del surgimiento de nuevos sujetos sociales, alerta contra la creciente criminalización de la disidencia, e insiste en la importancia de buscar las convergencias. Para finalizar este capítulo, Jorge Alonso pone en duda el propio término movimientos sociales, como invitación para que en la reflexión sobre cómo erradicar el capitalismo dicho concepto sea incluido, para encontrar otro más adecuado con las complejidades de nuestra América.

El apéndice es una reseña de Halloway (2011), libro que fue presentado en 2012 en la Universidad de Guadjara y que está relacionado con el resto del libro de Alonso, sobre 
Repensar los movimientos sociales

todo, con el tema de las convergencias. La frase que cierra este trabajo revela la preocupación del autor por encontrar salidas a la situación de un mundo cada día más injusto, más desigual, más indiferente a la condición humana; también, su búsqueda para que las convergencias se sitúen en el centro de la reflexión. Refiriéndose precisamente a ellas, Jorge Alonso escribe: "Sigo pensando que necesitamos muchas para salvarnos" (p. 124).

Repensar los movimientos sociales expone la complejidad y la dificultad de la respuesta a la pregunta cómo superar el capitalismo y su economía depredadora. Y tal complejidad incluye la visión del futuro: a qué sociedad se aspira y cómo alcanzarla. Más todavía: en qué tipo de relaciones sociales y actitudes culturales actuales podría localizarse el germen de la nueva convivencia. Quizá la conclusión más importante que alcanzamos al concluir la lectura sea que la esperanza del cambio está viva, aun cuando el propio término movimientos sociales parece ser insuficiente para aprehender la compleja realidad de nuestros días. La discusión de los puntos de vista de John Halloway es un complemento útil y exacto para pensar en la "grietas cotidianas" que se pueden abrir en el sistema. Alonso concuerda con esta posición que deja, a mi juicio, en una mala posición al leninismo. Son las convergencias de estas grietas, dice Jorge Alonso, las que a la larga constituyen el arma que terminará con el horror que hoy vivimos.

Halloway, John, 20I I, Agrietar el capitalismo, Ediciones HerraBibliografía mienta, Buenos Aires.

Marx, Carlos, 1968, "The British rule in India”, en Shlomo Avineri (ed.), Marx on colonialism and modernization, Penguin Books, Londres, pp. 88-96.

Nahim, Moisés , 20I4, El fin del poder, Randon House Mondadori, Barcelona. 